\title{
STRUCTURE AND PROPERTIES OF CARBON NANOTUBE REINFORCED NANOCOMPOSITES
}

\author{
Frank K. Ko, Sakina Khan, Ashraf Ali, Yury Gogotsi, Nevin Naguib \\ Guoliang Yang and Christopher Li \\ Drexel University, Philadelphia, PA, 19104 \\ Hideo Shimoda and Otto Zhou, \\ University of North Carolina, Chapel Hill, NC 27599 \\ Michael J. Bronikowski \\ Jet Propulsion Laboratory, California Institute of Technology, Pasadena, CA 91109 \\ Richard E. Smalley \\ Rice University, Houston, TX 77005; and \\ Peter A. Willis \\ Univ. of Calif., Los Angeles, CA 90095
}

\begin{abstract}
Carbon nanotubes (CNT) possess many unique characteristics that promise to revolutionize the world of structural materials resulting in significant impact on our capability to build lighter, smaller and higher performance structures for aerospace and many other industrial applications. When the CNT are aligned, micromechanical studies showed the potential of an order of magnitude increase in mechanical properties comparing to the state of the art carbon fiber reinforced composites. The coelectrospinning process is introduced as a pathway to realize this potential by aligning and carrying the CNT in the form of nanocomposite fibrils; thus forming the precursor for linear, planar and 3D fiber assemblies for macrocomposites. In this study, SWNT were purified and dispersed in polyacrylonitrile
\end{abstract}

solution for co-electrospinning into nanocomposite fibrils. The structure, composition and physical properties of these composite fibrils were characterized by Raman spectroscopy, TEM, AFM, and TGA.
Key words

Carbon nanotube, co-electrospinning, Raman spectroscopy, AFM, TEM, TGA

\section{Introduction}

Since the discovery of carbon nanotubes (CNT) by Iijima in 1991 [1], increasing attention has been attracted to this newly emerging material due to its remarkable mechanical and electrical properties [2, 3]. Based on its unique properties, many applications of CNT have been proposed including quantum wires, tiny electronic devices [4, 5], heterojunction devices, electron emitters [6], nanotube tips for scanning probe microscope [7], etc. One of the most intriguing applications of $\mathrm{CNT}$ is the polymer/CNT nanocomposites [8-13]. The superb mechanic property of CNT makes them ideal candidate as fillers in high strength, lightweight polymer composite. Polymers such as epoxy [10], thermoplastics [11], gels [12], as well as Poly (methyl methacrylate) (PMMA) [13] have been used as the matrix. However, the developments in polymer/CNT nanocomposite has been limited by the problems with the dispersion of the fillers as well as the load transfer across the CNT polymer interface due to the atomically smooth 
CNT surface. Well-aligned CNTs are also preferred since they are highly anisotropic. A number of methods have been used to achieve highly oriented CNT. [14-16]. All these methods are limited to microscale and are not suitable for commercial composite manufacturing process.

Based on a recent NASA study by Harris et al. [17] using micromechanics computation, it was concluded that an order of magnitude increase in specific modulus can be achieved with SWNT composites. Despite their promise, most current studies are limited to the physics and chemistry of individual SWNTs. There is limited knowledge on the properties of macroscopic materials comprising SWNTs as the basic building blocks for macroscopic structures. It is still not clear whether the superb properties observed at the individual molecular level can be scaled up to the macroscopic structures. In order to realize the exciting potential of SWNTs, there is a need for processing methodologies and robust manufacturing technologies to convert SWNTs to macroscopic structures. In a recent paper [ 18 ] by Ko et al, the concept of forma-

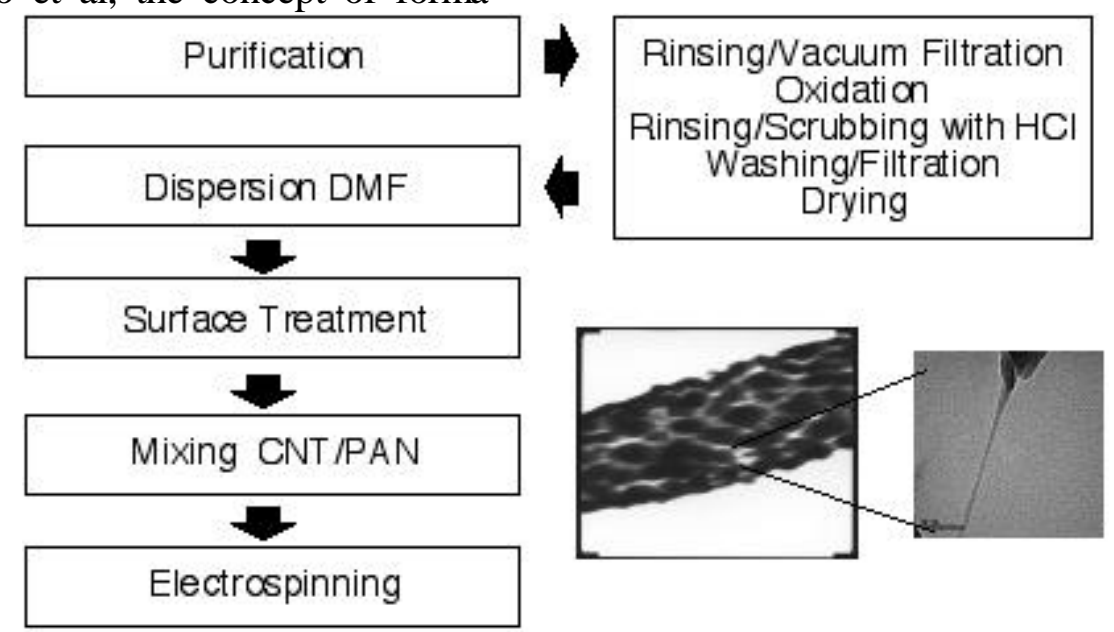

Figure 1. Processing Flow of SWNT/PAN Composite Fibrils and their TEM images. tion of super carbon nanotube fibril (SCF) by the electrospinning process was introduced. $\mathbf{h}$ this paper the HiPco single wall carbon nanotubes (SWNT) dispersed in a polyacrylonitrile matrix are used to demonstrate the validity of the SCF concept.

\section{Co-electrospinning of SWNT/PAN}

HiPco single wall nanotubes (SWNTs) obtained from Rice University, were purified, dispersed and mixed with Polyacrylonytrite (PAN) and coelectrospinning was done to produce the SWNT fibrils. The purification process for the HiPco SWNT is necessary because of the iron catalysts entrapped in carbon shells of the nanotubes must be removed. The purification process calls for multiple steps of oxidation, cleaning, rinsing and filtering according to the flow diagram shown in Fig. 1. In the same figure TEM images of the SWNT/PAN fibril are shown revealing a diamond pattern of the SWNT $s$. Some ligaments of the SWNT/PAN 5 $\mathrm{nm}$ in diameter were also observed.

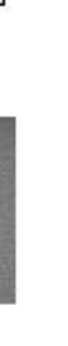


The electrospinning process is a nonmechanical method capable of the formation of nanoscale fibers electrostatically from polymer solutions or melts. Electrostatic generation of ultrafine fibers ("electrospinning") has been known since the 1930's [18,19] This technique has been recently rediscovered for applications such as high performance filters [21] and for scaffolds in tissue engineering [22] that utilize the unique characteristics of the high surface area $\left(\sim 10^{3}\right.$ $\mathrm{m}^{2} / \mathrm{gm}$ ) provided by the fibers. In this technique, a high electric field is generated between a polymer fluid contained in a glass syringe with a capillary tip and a metallic collection screen. When the voltage reaches a critical value, the charge overcomes the surface tension of the deformed drop of the suspended polymer solution formed on the tip of the syringe and a jet of ultrafine fibers is produced. The electrically charged jet

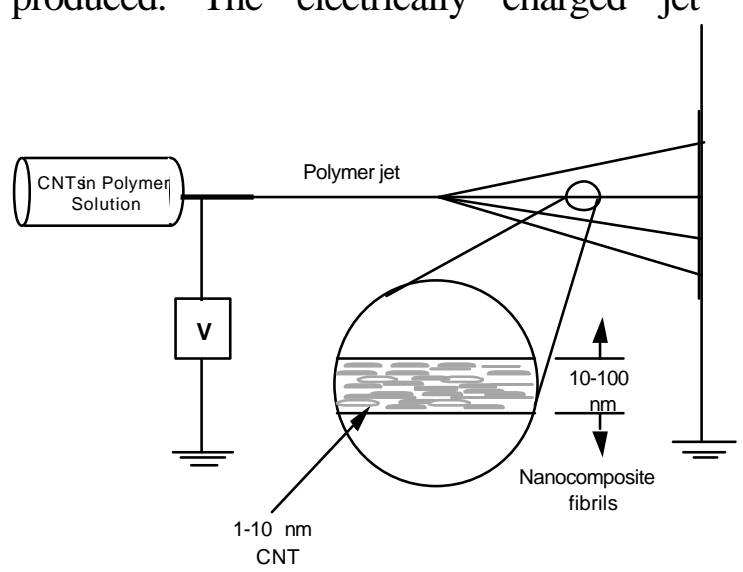

undergoes a series of electrically induced bending instabilities during its passage to the collection screen that results in the hyper-stretching of the jet. This stretching process is accompanied by the rapid evaporation of the solvent molecules that reduces the diameter of the jet in a cone shape radius. The dry fibers are accumulated on the surface of the collection screen or a collecting drum, resulting in a non-woven mat or linear assembly of nano to micron diameter fibers. The process can be adjusted to control the fiber diameter by varying the charge density $(\sim 1.5 \mathrm{KV} / \mathrm{cm}$ was employed) and polymer solution concentration, while the duration of electrospinning controls the thickness of fiber deposition. A schematic drawing of the electrospinning process of SWNT and PAN is shown in Figure 2. .

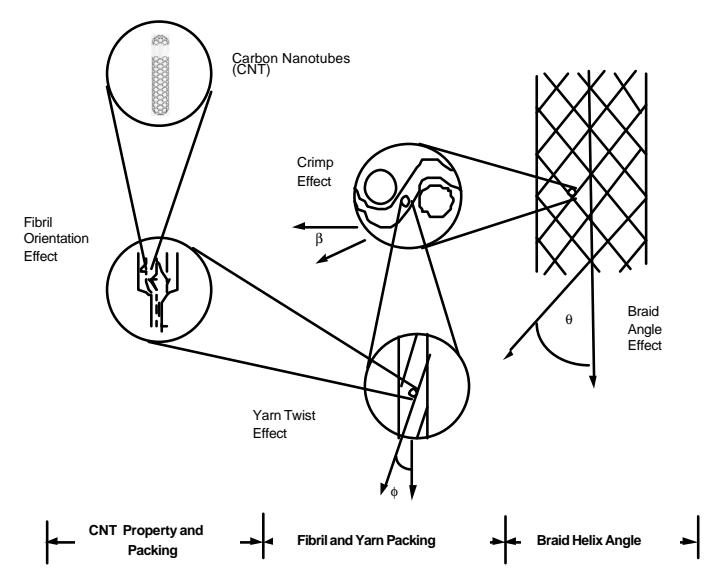

Figure 2. Co-electrospinning of SWNT/PAN Fibril and Fibrous Structures.

\section{Characterization of CNT Fibril}

\subsection{Raman Spectroscopy}

Raman spectroscopy analysis has been conducted on fiber spun with and without single wall nanotubes (SWNT) as shown in Figure 3. These spectra were recorded using a Renishaw Ramanmicrospectrometer Ramascope 1000 by using a diode laser (780nm excitation wavelength, $12 \mathrm{~W} / \mathrm{cm}^{2}$ ), which corresponds to the equivalent photon energy of $1.58 \mathrm{eV}$. Typical peaks of SWNT's can be seen in the fiber spun with nanotubes, which serves as a direct confirmation of the successful filling of polymers with the nanotubes. 
The most intense bands in the Raman spectra of single wall carbon nanotubes are the radial breathing mode (RBM) near $180 \mathrm{~cm}^{-1}$ and the modes between $1500-1600 \mathrm{~cm}^{-1}$ associated with the tangential displacement of $\mathrm{C}-\mathrm{C}$ bond stretching motions of the nanotubes. RBM belongs to the identity representation $\left(A_{1 g}\right.$ or $A_{1}$ radial band), while 1500 $1600 \mathrm{~cm}^{-1}$ range belongs to $E_{1 \mathrm{~g}}$ or $\mathrm{E}_{2 \mathrm{~g}}$ symmetry (tangential band) [23]

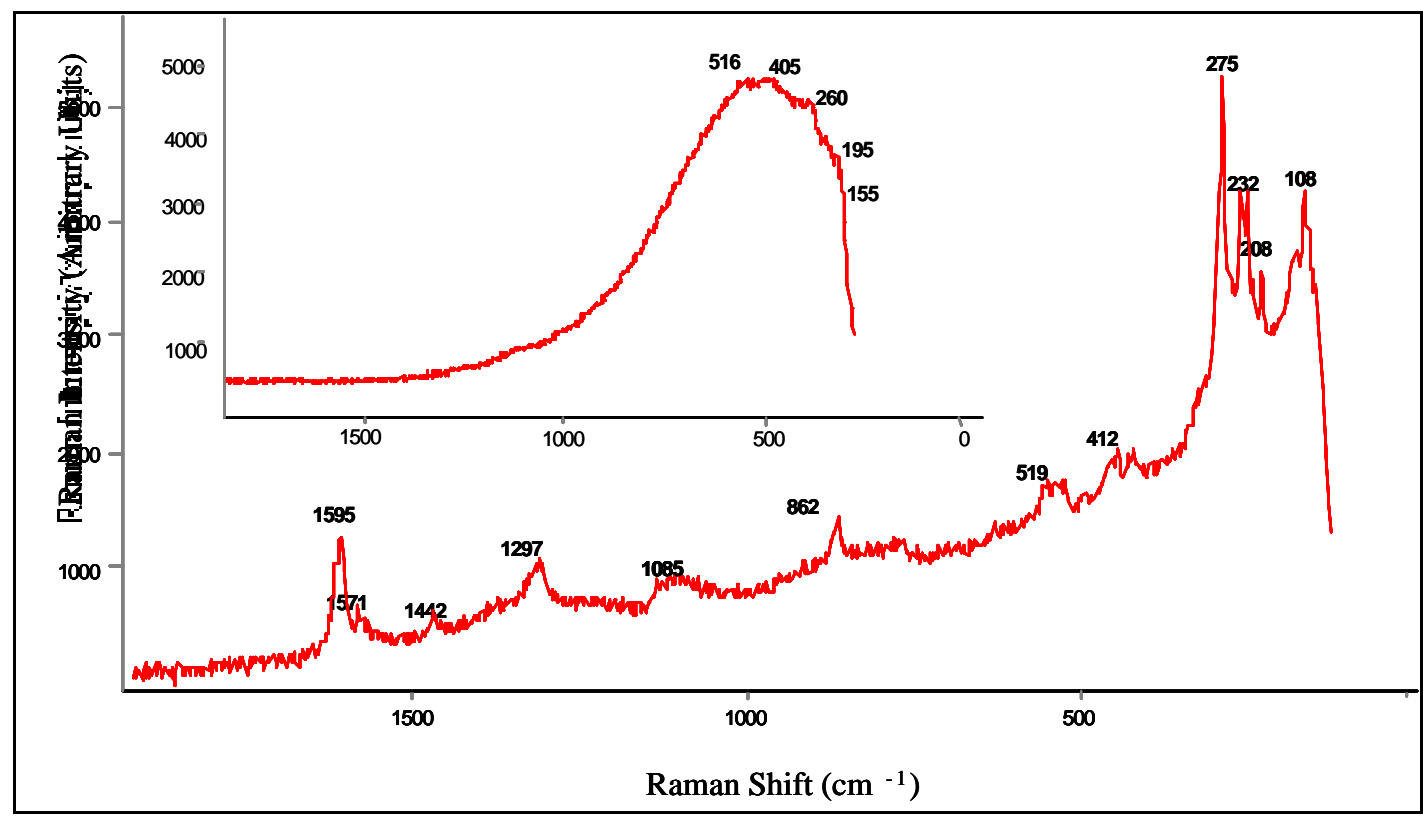

Figure 3. Raman Spectra of PAN and SWNT/PAN at $780 \mathrm{~nm}$ Excitation

According to the theoretical predictions $\mathrm{RBM}$ frequency is inversely proportional to the tube diameter, without any dependence on the chiral angle. On the other hand, it has been also shown that the frequencies and the number of the Raman active modes between 1500$1600 \mathrm{~cm}^{-1}$ depend on the diameter and the chirality of the nanotube. However, within each type of nanotube, the modes in the $1580-1590 \mathrm{~cm}^{-1}$ range are expected to be very weakly dependent on the nanotube diameter, by perhaps an order of magnitude less than for the case of the diameter dependence of the RBM.

From the results shown here, the most intense band in the Raman Spectra of SWNT-filled fiber is the radial breathing mode (RBM) near $270 \mathrm{~cm}^{-1}$. The presence of at least $6 \mathrm{RBM}$ peaks is ob- served in the range from130-275 $\mathrm{cm}^{-1}$. This corresponds to a very large diameter distribution shown by the lowfrequency bands and confirmed by the broader splitting of the $\mathrm{E}_{2 \mathrm{~g} 2}$ graphite mode, which simply means that the sample has different tube diameters.

\subsection{Thermal Stability by Thermal Gravimetric Analysis}

The CNT contents in the PAN nanofibers can be detected by the thermogravimetry method. Both pure PAN $r$ and PAN-CNT nanofibers were heated in the air atmosphere at a rate of $20^{\circ} \mathrm{C} / \mathrm{min}$. Decomposition of PAN takes place at $308^{\circ} \mathrm{C}$ for the pure PAN nanofiber and $325^{\circ} \mathrm{C}$ for the PAN-CNT nanofiber, $\dot{n}-$ 
dicating that $\mathrm{CNT}$ in the PAN nanofibers increases PAN's thermal stability. The difference of the remaining weight between these two samples $(56.8 \%$ for pure PAN and $61.2 \%$ for PAN-CNT) at the plateau region (around $330^{\circ} \mathrm{C}$ ) is due to the CNT contents in the PAN-CNT nanofibers. Assuming that the same decomposition mechanism for these two samples, (thermal pyrolysis of PAN is relatively complicated; since our main goal is to determine the CNT contents in the nanofibers, the detailed mechanism will not be discussed here)[24,25] the CNT content in the PAN-CNT can therefore be calculated to be $10 \mathrm{wt} \%$. The results suggest that $10 \%$ of CNT in the PAN-CNT nanofibers increases the decomposition temperature of PAN nanofibers by $17^{\circ} \mathrm{C}$.

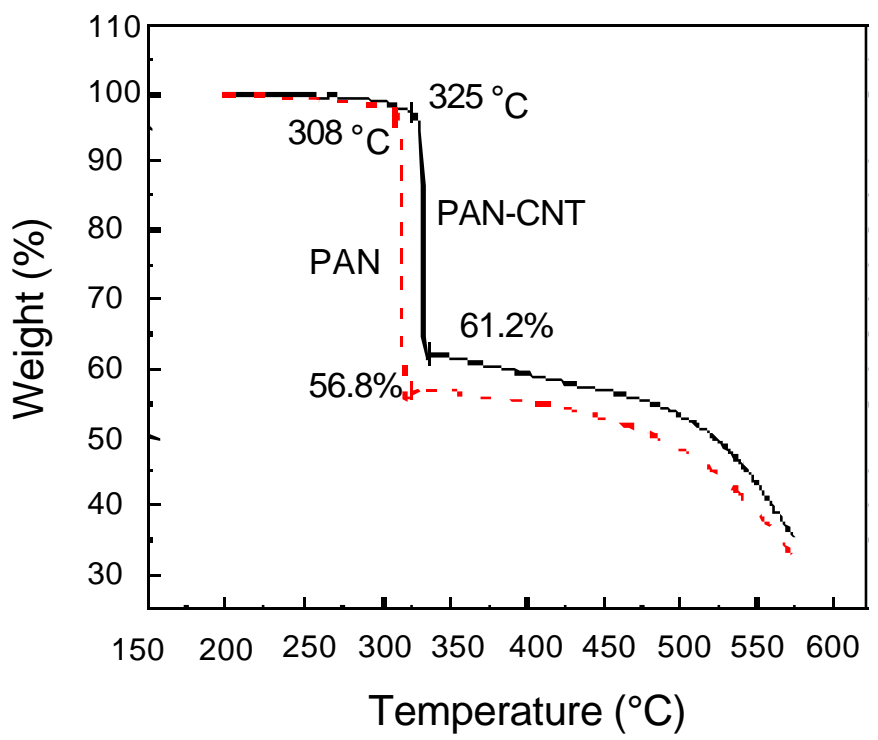

Figure 4. TGA analysis of PAN (dotted line) and PAN-CNT (solid line) nanofibers in air.

\subsection{Atomic Force Microscopy}

The atomic force microscope (AFM) offers the opportunity not only to characterize the surface topography of the fibers but also to investigate their mechanical, electrical and thermal properties, with the utilization of appropriate probes. Since the structural, mechanical, electrical and thermal properties of the SWNT/PAN fibrils are expected to be highly anisotropic and heterogeneous, the high spatial resolution of AFM makes it an ideal tool to measure these properties locally. These local properties will reveal certain microscopic mechanisms for the observed behaviors of these fibers on the macroscopic scale.

Surface structure characterization - Both tapping-mode and contactmode AFM imaging will be used to characterize the surface structures of the SWNT/PAN fibers. AFM imaging requires a flat substrate and a sparse distribution of fibers firmly attached to the substrate. We have found hat depositing the fibers directly on a fresh mica surface during the electrospinning produces satisfactory specimens for AFM imaging. The 
attachment of the fibers to the mica substrate is strong enough to withstand imaging. Preliminary results show that PAN fibers and the SWNT/PAN fila- ments have distinctly different surface structures, as shown in figures $5 \mathrm{a}$ and $5 b$.

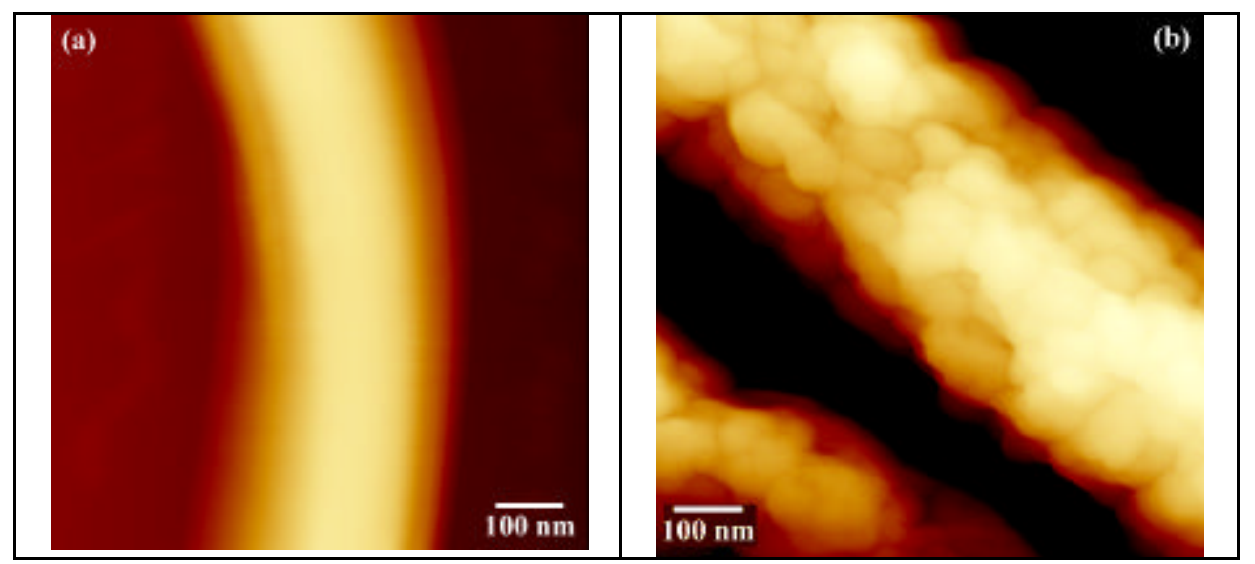

Figure 5. AFM images of (a) PAN fiber; (b) SWNT/PAN Fibril

The questions that can be addressed ising AFM imaging include how the inclusion of SWNTs changes the surface morphology of the fiber, how the SWNTs are oriented in the fibers, and how the fibers interact with each other at the crossover points. Since AFM can image samples submerged in liquids, the effects of different solvents

on the fiber properties can be monitored and measured.

\section{Mechanical properties meas-} urements - The AFM has been used to investigate the mechanical properties of carbon nanotubes [26-29]. We have used this approach to measure the mechanical properties of the SWNT/PAN fibers. In such an experiment, the fibers are first imaged, and the AFM tip is then positioned on top of the fiber at the point of interest, and the sample surface is then raised as shown in figure 6(a).
The force is obtained from the deflection of the cantilever $\Delta \mathrm{d}$, the amount of fiber indentation/deformation $\Delta \mathrm{z}$ can be obtained from the difference of the sample height increase $\Delta \mathrm{h}$ and $\Delta \mathrm{d}: \Delta \mathrm{z}=\Delta \mathrm{h}-\Delta \mathrm{d}$. The elastic modulus of the fiber can be evaluated based on these measured parameters using the approach of Kracke and Damaschke [27]. The method utilizes the relationship $\mathrm{dF} / \mathrm{d}(\Delta \mathrm{z})=$ $\left(2 / \pi^{1 / 2}\right) E^{*} A^{1 / 2}$, where $A$ is the contact area, $E^{*}$ is the effective Young's modulus of the contact as defined by: $1 / \mathrm{E}^{*}=\left(1-\mathrm{v}_{1}^{2}\right) / \mathrm{E}_{1}+\left(1-\mathrm{v}_{2}^{2}\right) / \mathrm{E}_{2}$. Here, $\mathrm{E}_{1}$, $E_{2}$ and $v_{1}$ and $v_{2}$ are the elastic moduli and the Poisson's ratios of the sample and the tip. Figures 6(b) and 6(c) show the results of mechanical property measurements on SWNT/PAN and PAN fibers. Mica was used as a standard since its elastic modulus has been determined the same method [27]. 
(a)
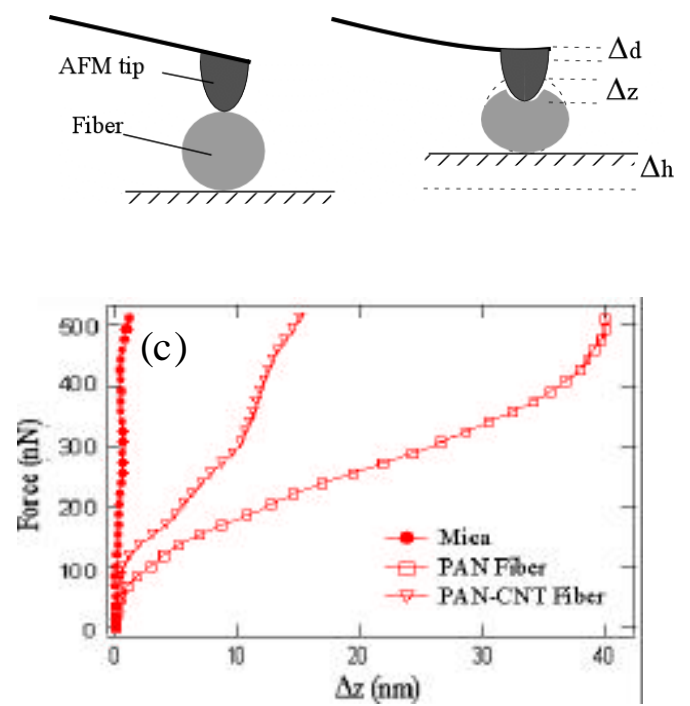

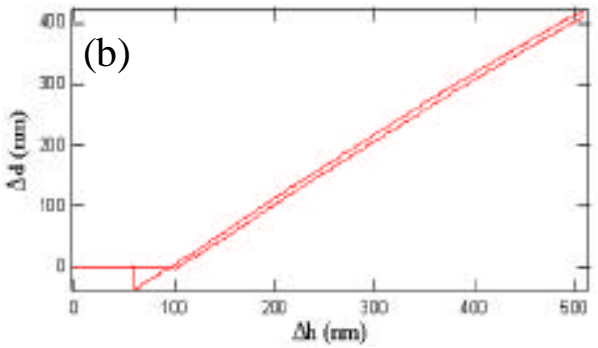

Figure 6. (a) Schematic for elasticity measurement. (b) Cantilever deflection vs. sample height in a cycle of compression and relaxation. (c) Load vs. indentation curves

The fibrils show a non-linear behavior in the load versus indentation plot, as shown in figure 6(c). The cause of this non-linear behavior of the loaddeformation curves is still under investigation. Using the first linear region in the plot $(125 \mathrm{nN}-300 \mathrm{nN})$, the Young's modulus of the SWNT/PAN fibril has been estimated to be $\sim 2.0 \mathrm{GPa}$, while that of the PAN fibrils has an approximate value of $\sim 1.0 \mathrm{GPa}$. Although there is still a long way from realizing the theoretical SWNT/PAN composite modulus of $100 \mathrm{GPa}$, considering the tensile modulus of the unfilled PAN nanofibers of $\sim 1 \mathrm{GPa}$, the reinforcement effect is quite remarkable with only less than $10 \%$ weight of SWNT was added showing a ductile mode of failure. For data showing in figure 6 , a silicon nitride cantilever with a spring constant of 0.58 $\mathrm{N} / \mathrm{m}$ was used. Each curve in figure $6(\mathrm{c})$ is the average of several curves measured on the same material. The values of tip parameters used in the estimation of the elastic modulus are: $v_{2}=0.27$,
$\mathrm{E}_{2}=130 \mathrm{GPa}$ for the radius of contact area $=5.0 \mathrm{~nm}$.

\section{Summary and Conclusions}

Co-electrospinning process is a viable means to produce continuous polymeric filaments filled with SWNT. Purified HiPco SWNT were dispersed in PAN solution and co-electrospun achieving a weight fraction approaching 10\%. Raman spectroscopy and TEM images showed convincingly the presence of SWNT in the electrospun PAN fibrils. AFM images showed a rough surface of the SWNT/PAN fibril as compared to the smooth surface appearance of the PAN fibrils. TEM images further confirm the presence of SWNT along the length of the fibrils revealing a diamond pattern of the SWNTsThe thermal stability of the SWNT/PAN was demonstrated by TGA showing greater than $15^{\circ} \mathrm{C}$ increase in decomposition temperature comparing to PAN. Load-deformation relationship of single SWNT/PAN fibril was characterized showing a ductile 
mode of failure and a strong reinforcement effect by doubling the tensile modulus with less than $10 \%$ reinforcement by weight.

\section{Acknowledgements}

This work was partially supported through NASA grant NAG-101061 (Ko and Zhou). Funding from NASA, ONR and the Texas Advanced Technology program to the Smalley group is greatly appreciated.

\section{$\underline{\text { 6. References }}$}

1. D. A. Walters, L. M. Ericson, M. J. Casavant, J. Liu, D. T. Colbert, K. A. Smith, and R. E. Smalley, Elastic strain of freely suspended single-wall carbon nanotube ropes, Appl. Phys. Lett., 74, 3803-3805(1999),

2. H. Ishikawa, S. Fudetani and M. Hrohashi, Mechanical properties of thin films measured by nanoindenters, Appl. Surf. Sci. 178, 5642(2001).

3. B. Kracke and B. Damaschke, Measurement of nanohardness and nanoelasticity of thin gold films with scanning force microscope, Appl. Phys. Lett. 77, 361-363(2000).

4. T. Hertel, R. Martel and P. Avouris, Manipulation of Individual Carbon Nanotubes and Their Interaction with Surfaces, J. Phys. Chem. B 102, 910915(1998).

5. H. Dai, E.W. Wong and C. M. Lieber, Probing Electrical Transport in Nanomaterials: Conductivity of Individual Carbon Nanotubes, Science, 272, 523-526(1996).

6. T. Tombler, C. Zhou, L. Alexseyev, J. Kong, H. Dai, L. Liu, C. S. Jayanthi, M. Tang and $\mathrm{S}$. Wu, Reversible electromechanical characterization of carbon nanotubes under local-probe manipulation. Nature, 405, 769772(2000).

7. P.J. de Pablo, M.T. Martinez, J. Colchero, J. Gomez-Herrero, W.K. Maser, A.M. de Benito, E. Munoz and A.M. Baro, Electrical characterization of single-walled carbon nanotubes with Scanning Force Microscopy, Mater.Sci. Eng. C15, 149151(2001).

8. L. Cai, H. Tabata and T. Kawai, Self-assembled DNA networks and their electrical conductivity, Appl. Phys. Lett. 77, 3105-3106(2000).

9. M.C. Hersam, A.C. F. Hoole, S.J. O'Shea, and M.E. Welland, Potentiometry and repair of electrically stressed nanowires using atomic force microscopy, Appl. Phys. Lett. 72, 915-917(1998).

10. Facundo Ruiz, W. D. Sun, and Fred H. Pollak and Chandra Venkatraman, Determination of the thermal conductivity of diamond-like nanocomposite films using a scanning thermal microscope, Appl. Phys. Lett. 73, 1802-1804(1998).

11. G.B.M. Fiege, A. Altes, R. Heiderhoff and L.J. Balk, Quantitative thermal conductivity measurements with nanometre resolution, J. Phys. D 32, L13-L17(1999).

12. S. Gomes, N. Trannoy and P. Grossel, DC thermal microscopy: study of the thermal exchange between a probe and a sample, Meas. Sci. Technol. 10, 805-811(1999).

13. O. Laurie, D.E. Cox, and H.D. Wagner. Bucking and collapse of embedded carbon nanotubes. Appl Phys. Lett., 81(8):1638-1641,. 1998.

14. C. Bower, R. Rosen, L. Jin, J. Han, and 0. Zhou. Deformation of carbon nanotubes in nanotubular polymer composites. Phys. Lett., 74(22):3317-3319, 1999. 
15. L.S. Schadler, S.C. Giannaris, and P.M. Ajayan. Appl Phys. Lett., 73:3842-3844, 1999.

16. L. Jin, C. Bower, and O. Zhou, Alignment of carbon nanotubes in a polymer matrix by mechanical stretching. Appl. Phys. Lett., 73(9):1197-1199, 1998.

17. C.E. Harris, J.H. Starnes, Jr., and M.J. Shuart, An Assessment of the State of the Art in the Design and Manufacturing of Large Composite Structures for Aerospace vehicles, NASA/TM-2001-210844, April 2001

18. F.K. Ko W. B. Han , A. Rahman, H. Shimoda and O. Zhou , Carbon Nanotube Reinforced Nanocomposites by the Electrospinning Process, Proceedings, Am. Soc. for Comp., Virginia Poly. Tech. Inst. September 2001.

19. A. Formhals, US Patent $1,975,504$, 1934

20. J. Doshi and D.H. Reneker, Journal of Electrostatics, 35 (1995) 151.

21. P.W. Gibson, H.L. Schreuder-Gibson and D. Riven, AIChE Journal, 45 (1999) 190.

22. F.K. Ko, C.T. Laurencin, M.D. Borden and D.H. Reneker, "The Dynamics of Cell-Fiber Architecture Interaction", Proceedings, Annual Meeting, Biomaterials Research Society, San Diego, April, 1998.
23. W.H. Weber, R. Merlin. Raman Scattering in Materials Science. Springer-Verlag Berlin Heildberg 2000.

24. W. J. Burlant and J. L. Parsons Pyrolysis of Polyacrylonitrile, J. Polym. Sci. XXII, 249-256(1956).

25. J. E. Bailey and A. J. Clarke Carbon Fibre Formation - the Oxidation Treatment, Nature, 234, 529531(1971).

26. D. A. Walters, L. M. Ericson, M. J. Casavant, J. Liu, D. T. Colbert, K. A. Smith, and R. E. Smalley, Elastic strain of freely suspended single-wall carbon nanotube ropes, Appl. Phys. Lett., 74, 3803-3805(1999),

27. H. Ishikawa, S. Fudetani and M. Hrohashi, Mechanical properties of thin films measured by nanoindenters, Appl. Surf. Sci. 178, 5642(2001).

28. B. Kracke and B. Damaschke, Measurement of nanohardness and nanoelasticity of thin gold films with scanning force microscope, Appl. Phys. Lett. 77, 361-363(2000).

29. T. Hertel, R. Martel and P. Avouris, Manipulation of Individual Carbon Nanotubes and Their Interaction with Surfaces, J. Phys. Chem. B 102, 910915(1998). 